This is a postprint version of the following published document:

Lo, M. C., Guzmán, R., Ali, M., Santos, R., Augustin, L. \& Carpintero, G. (2107). 1.8-THz-wide optical frequency comb emitted from monolithic passively mode-locked semiconductor quantum-well laser. Optics Letters, 42 (19), pp. 3872-3875.

DOI: $10.1364 / \mathrm{OL} .42 .003872$

(C) Optical Society of America (OSA), 2017 


\title{
1.8-THz-wide optical frequency comb emitted from monolithic passively mode-locked semiconductor quantum-well laser
}

\author{
Mu-Chieh Lo, ${ }^{1, *}$ Robinson Guzmán, ${ }^{1}$ Muhsin Ali, ${ }^{1}$ Rui Santos, ${ }^{2,3}$ Luc Augustin, ${ }^{2,3}$ \\ and Guillermo Carpintero ${ }^{1}$ \\ ${ }^{1}$ Universidad Carlos III de Madrid, Av. Universidad 30, 28911 Leganés, Spain \\ ${ }^{2}$ COBRA Research Institute, Technische Universiteit Eindhoven, 5600 MB Eindhoven, The Netherlands \\ ${ }^{3}$ SMART Photonics, Horsten 1, 5612 AX Eindhoven, The Netherlands \\ *Corresponding author: mlo@ing.uc3m.es
}

\begin{abstract}
We report on an optical frequency comb with $14 \mathrm{~nm}(\sim 1.8 \mathrm{THz})$ spectral bandwidth at a $-3 \mathrm{~dB}$ level that is gen-erated using a passively mode-locked quantum-well laser in photonic integrated circuits fabricated through an InP generic photonic integration technology platform. This $21.5-\mathrm{GHz}$ colliding-pulse mode-locked laser cavity is de-fined by on-chip reflectors incorporating intracavity phase modulators followed by an extracavity semiconductor opti-cal amplifier as a booster amplifier. A 1.8-THz-wide optical comb spectrum is presented with an ultrafast pulse that is $0.35 \mathrm{ps}$ wide. The radio frequency beat note has a $3-\mathrm{dB}$ line-width of $450 \mathrm{kHz}$ and $35-\mathrm{dB}$ SNR.
\end{abstract}

OCIS codes:

(140.4050) Mode-locked lasers; (140.5960) Semiconductor lasers; (250.5300) Photonic integrated circuits.

Integrated passively mode-locked semiconductor lasers are robust and compact sources for generating coherent optical frequency combs, which are of great interest in communications, metrology, spectroscopy, and millimeter wave/terahertz $(\mathrm{mmW} / \mathrm{THz})$ generation [1]. Unlike other optical frequency comb generation techniques, it does not require an external radio frequency $(\mathrm{RF})$ source or optical pump, although it can be synchronized to an external RF reference to decrease noise. A passively mode-locked laser is usually a two-section structure, composed of a semiconductor optical amplifier (SOA) as the gain section and a saturable absorber (SA) in a Fabry-Perot cavity [2]. Alternatively, a passively mode-locked laser can be designed in such a way that SAs are placed at certain positions within the resonator to support multiple pulses that meet in the absorbers, which is the colliding-pulse mode-locking scheme [3]. When developed in active-passive integration, passive waveguide components, namely, multimode interference couplers/ reflectors (MMIs/MIRs), electro-optic phase modulators (EOPMs) are enabled to include more functionality and design freedom [4]. The use of passive waveguides enables SOA and SA lengths to be optimized, thus reducing the self-phase modulation in active media [5,6]. Using MMIs and curved waveguides, modelocked lasers in ring geometry are feasible [7,8]. MIRs allow for an on-chip Fabry-Perot cavity [9], signal acquisition, and poststage processing [10]. In [11-13], it was shown that by introducing a phase-shifter-based intracavity gain-flattening filter, the optical bandwidth, which is the key characteristic of a comb, can be significantly improved.
Recent studies on the bandwidth of a comb generated from a passively mode-locked semiconductor laser have revealed that the performance of quantum-dash (Q-dash) and quantum-dot (QD) active regions is better than that of quantum-well (QW) active regions [14,15]. A QD-based passively mode-locked laser with a $12-\mathrm{nm}, 3-\mathrm{dB}$ optical bandwidth was presented in [16]. A frequency comb of more than $16 \mathrm{~nm}$ was demonstrated from a Q-dash laser in [17]. In [18], a 3-dB optical bandwidth of $11.5 \mathrm{~nm}$ was reached with a $20-\mathrm{GHz}$ ring passively modelocked laser, which was a record value for QW. In [19], the same group proposed another design using the same technology to generate a wide comb with a $42-\mathrm{nm}, 20-\mathrm{dB}$ bandwidth.

In this Letter, we present an integrated mode-locked QW laser developed through a generic InP photonic integration platform with a new record bandwidth of $14 \mathrm{~nm}$ that is comparably wider than that of a passively mode-locked QD-based laser. The proposed design is a colliding-pulse mode-locked laser in a symmetric arrangement. New features that we have included in this device are symmetrically positioned intracavity electro-optic phase modulators (EOPMs) and an extracavity SOA. The device is demonstrated to generate an extremely broad optical spectrum that constitutes an optical frequency comb. The phase locking of modes agrees with the autocorrelation trace, showing ultrafast pulses. The optical comb has a record 3-dB bandwidth of $14 \mathrm{~nm}(1.8 \mathrm{THz})$, even wider than that of a QD-based passively mode-locked laser.

Figure 1(a) depicts the proposed photonic integrated circuit (PIC) microscope photo. Figure 1(b) presents the schematic. 


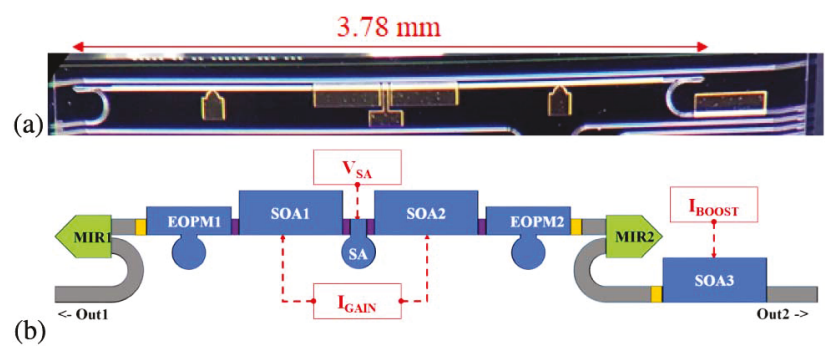

Fig. 1. (a) Micrograph. (b) Schematic. SA, saturable absorber; SOA, semiconductor optical amplifier; EOPM, electro-optic phase modulator.

This PIC was developed through a generic approach and fabricated within a multi-project wafer (MPW) run by SMART Photonics [20]. This platform uses ridge waveguides that are $1.5 \mu \mathrm{m}$ (deeply etched) and $2.0 \mu \mathrm{m}$ (shallowly etched) wide. The stack has a 500 -nm-thick InGaAsP $Q=1.25$ layer, and the active waveguides consist of four quantum wells. The process design kit (PDK) provides a selection of predefined optoelectronic components as parametric building blocks [21]. The PIC is composed of one SA, two SOAs, two EOPMs, two MIRs, and straight/bent passive waveguides in a symmetric geometry with respect to the SA. Without using cleaved facets as reflectors, lithographically defined MIRs assure a reliable and reproducible operation [22], and the total cavity length defined by the MIRs is $3.78 \mathrm{~mm}$, corresponding to a repetition rate of about $21.5 \mathrm{GHz}$. This $30-\mu \mathrm{m} \mathrm{SA}$ is surrounded by two 400- $\mu \mathrm{m}$ SOAs with two $1200-\mu \mathrm{m}$-long EOPMs on both sides. Between active components, isolations were placed to avoid unwanted current flows. Transitions were inserted between the deeply etched and shallowly etched waveguides. The two MIRs have $50 \%$ reflectivity and $50 \%$ transmissivity on two ports. In both MIRs, one port connects to the active components and forms a linear resonant cavity. The other port either sends out the pulse train directly to the facet edge through the left $7^{\circ}$ angled waveguide (OUT1) or to the other $7^{\circ}$ angled waveguide (OUT2) via the $400-\mu \mathrm{m}$ booster amplifier (SOA3). The deeply etched waveguides have a width of $1.5 \mu \mathrm{m}$ and a bending radius of $100 \mu \mathrm{m}$. Each active component was with a metal pad for electrical contact.

For electrically driving the PIC, the metal pads of the PIC were wire-bonded and connected to external DC sources as shown in Fig. 1(b). The PIC was biased with voltage sources (Agilent E3634A Power Supplies) and current sources (Thorlabs PRO8000 LD Controller), denoted as $V_{\mathrm{SA}}, I_{\mathrm{GAIN}}$,

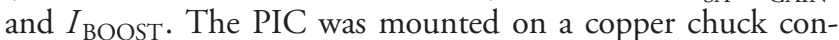
trolled at $16^{\circ} \mathrm{C}$ with a Thorlabs PRO8000 TEC Controller. The optical output signal from OUT2 was coupled to an $\mathrm{Oz}$ Optics lensed fiber. The collected optical signal was diagnosed on a Thorlabs power meter (PM), Yokogawa AQ6370B optical spectrum analyzer (OSA), APE PulseCheck autocorrelator (AC), and Anritsu MS2668C electrical spectrum analyzer (ESA). We used an Amonics erbium-doped fiber amplifier (EDFA) to pump the power up to $10 \mathrm{dBm}$ and a polarization controller (PC) prior to the autocorrelator. For the electrical beat tone measurement, a Nortel EDFA with $10 \mathrm{dBm}$ output power and an $u^{2} t 40-\mathrm{GHz}$ photodiode (PD) were employed.

The influence on $P_{\text {Out2 }}$ (optical power collected at Out2) of $I_{\text {GAIN }}$ and $I_{\text {BOOST }}$ are shown in Fig. 2(a) and 2(b), respectively. (a)

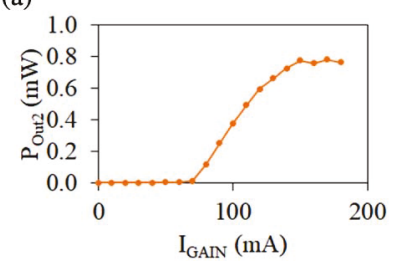

(b)

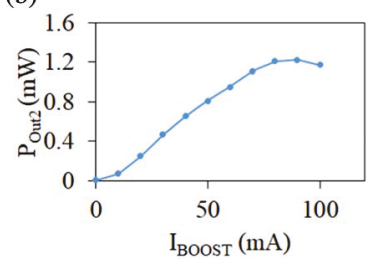

Fig. 2. Light-current characteristics: (a) $P_{\text {Out2 }}$ versus $I_{\text {GAIN }}$ when $V_{\text {SA }}=0 \mathrm{~V}$ and $I_{\text {BOOST }}=50 \mathrm{~mA}$; (b) $P_{\text {Out2 }}$ versus $I_{\text {BOOST }}$ when $V_{\mathrm{SA}}=0 \mathrm{~V}$ and $I_{\mathrm{GAIN}}=160 \mathrm{~mA}$.

In Fig. 2(a), as $V_{\mathrm{SA}}=0 \mathrm{~V}$ and $I_{\mathrm{BOOST}}=50 \mathrm{~mA}$ with $I_{\mathrm{GAIN}}$ varied from 0 to $200 \mathrm{~mA}$, the L-I curve exhibits a typical laser characteristic with threshold current of $I_{\text {GAIN }}<80 \mathrm{~mA}$, and the slope efficiency is around $10 \mathrm{~mW} / \mathrm{A}$. In Fig. 2(b), as $V_{\mathrm{SA}}=0 \mathrm{~V}$ and $I_{\text {GAIN }}=160 \mathrm{~mA}$ with $I_{\mathrm{BOOST}}$ varied from 0 to $100 \mathrm{~mA}$, the L-l curve is showing a nearly linear trend with a $15-\mathrm{mW} / \mathrm{A}$ slope efficiency, and it enters the saturation region when $I_{\text {BOOST }}>80 \mathrm{~mA}$. The PIC can emit more than $1 \mathrm{~mW}$ optical power as the booster amplifier SOA3 is fed with sufficient bias current.

The optical spectra at various bias conditions are plotted in Figs. 3(a) and 3(b), where the influences of $V_{\mathrm{SA}}$ and $I_{\mathrm{GAIN}}$ are visualized. Under the bias condition of $V_{\mathrm{SA}}=0 \mathrm{~V}$, $I_{\mathrm{BOOST}}=30 \mathrm{~mA}$, and $I_{\text {GAIN }}$ swept from 80 to $200 \mathrm{~mA}$, the optical spectra are shown in Fig. 3(a), while those at $V_{\mathrm{SA}}=-0.6 \mathrm{~V}, I_{\mathrm{BOOST}}=30 \mathrm{~mA}$, and $I_{\mathrm{GAIN}}$ swept from 80 to $200 \mathrm{~mA}$ are shown in Fig. 3(b). The only different parameter between Figs. 3(a) and 3(b) is $V_{\mathrm{SA}}$. In Fig. 3(a), as $I_{\mathrm{GAIN}}$ increases, the lasing wavelength shifts upward, and the profile becomes broader. In Fig. 3(b), similarly, the lasing position moves upward when $I_{\mathrm{GAIN}} \leq 120 \mathrm{~mA}$. There is a wavelength jump from $1555 \mathrm{~nm}$ to $1535 \mathrm{~nm}$ between $I_{\text {GAIN }}=120 \mathrm{~mA}$ and $I_{\mathrm{GAIN}}=140 \mathrm{~mA}$. When $I_{\mathrm{GAIN}} \geq 140 \mathrm{~mA}$, the wide modelocking spectra are formed at about $1535 \mathrm{~nm}$. The optical spectrum is changed not only with $V_{\mathrm{SA}}$ and $I_{\mathrm{GAIN}}$ but also $I_{\mathrm{BOOST}}$. In Fig. 3(c), a series of optical spectra with different $I_{\mathrm{BOOST}}$ are (a)

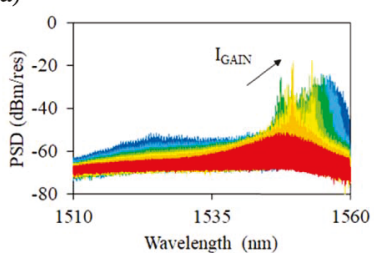

(b)

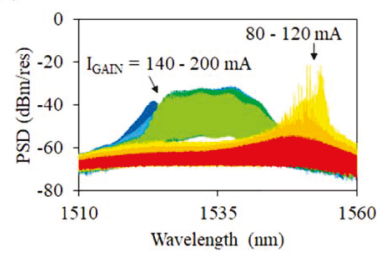

(c)

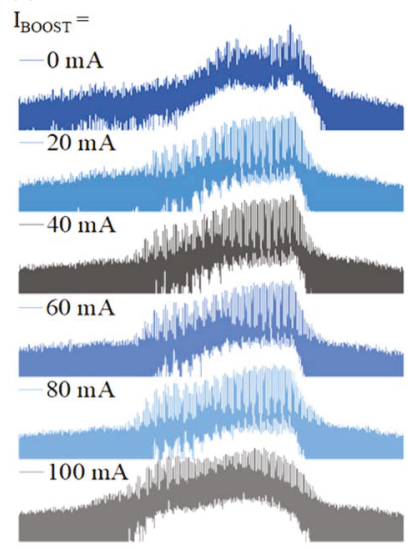

Fig. 3. (a) Optical spectra at Out2, $V_{\mathrm{SA}}=0 \mathrm{~V}$, $I_{\mathrm{BOOST}}=30 \mathrm{~mA}, I_{\mathrm{GAIN}}=80-200 \mathrm{~mA}$. (b) Optical spectra at Out2, $V_{\mathrm{SA}}=-0.6 \mathrm{~V}, \quad I_{\mathrm{BOOST}}=30 \mathrm{~mA}, I_{\mathrm{GAIN}}=80-200 \mathrm{~mA}$. (c) Optical spectra at Out2, $V_{\mathrm{SA}}=-0.6 \mathrm{~V}, I_{\mathrm{BOOST}}=0-100 \mathrm{~mA}$, $I_{\mathrm{GAIN}}=150 \mathrm{~mA}$. 
(a)

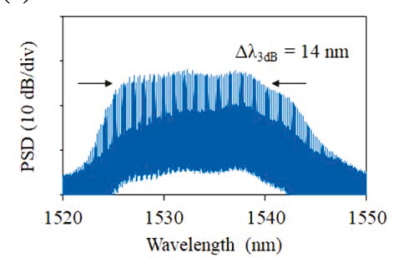

(b)

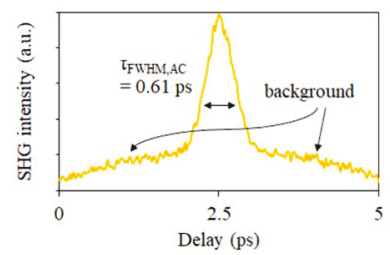

Fig. 4. At Out2, $V_{\mathrm{SA}}=-0.6 \mathrm{~V}, \quad I_{\mathrm{GAIN}}=180 \mathrm{~mA}$, $I_{\mathrm{BOOST}}=30 \mathrm{~mA}$. (a) Optical spectrum. Resolution bandwidth: $0.02 \mathrm{~nm}$. 3-dB bandwidth $=14 \mathrm{~nm}(1.8 \mathrm{THz})$. SMSR $\sim 8 \mathrm{~dB}$. (b) Autocorrelation trace. FWHM of AC trace $\left(\tau_{\mathrm{FWHM}, \mathrm{AC}}\right)=$ 0.61 ps. Assuming a sech ${ }^{2}$ pulse shape, $\tau_{\mathrm{FWHM}, \mathrm{SECH} 2}=0.35$ ps.

depicted. When $V_{\mathrm{SA}}=-0.6 \mathrm{~V}$ and $I_{\mathrm{GAIN}}=150 \mathrm{~mA}$, the optical spectrum gradually gets broader and flattened as $I_{\text {BOOST }}$ increases. However, the suppression ratio does not monotonically depend on $I_{\text {BOOST }}$.

The frequency comb generated from the PIC is demonstrated in Fig. 5(a) with a resolution bandwidth of $0.02 \mathrm{~nm}$. It exhibits an extremely broad spectrum with maximum level at $1537.44 \mathrm{~nm},-32.119 \mathrm{dBm} /$ res, and the spectral width at $3-\mathrm{dB}$ level is $14 \mathrm{~nm}$. Within the $3-\mathrm{dB}$ bandwidth, it covers 83 comb teeth, and the mode spacing is about $0.172 \mathrm{~nm}$ corresponding to $21.5 \mathrm{GHz}$. The side-mode suppression ratio (SMSR) is $8 \mathrm{~dB}$, which is relatively weak. The wide bandwidth leads to a very short temporal trace, as shown in Fig. 4(b), where a pulse appears at the midpoint of the time delay span. The full width at half-maximum (FWHM) of the AC trace is 0.61 ps. The FWHM of the pulse, assuming a sech ${ }^{2}$ shape, is 0.35 ps. The time-bandwidth product (TBP) is 1.01 , not close enough to the transform limit 0.315 , indicating that this pulse is chirped and can be further compressed. It should be noted that the pulse is standing on a pedestal. It may be attributed to the complicated intensities with slowly varying envelopes, and the non-zero low-frequency amplified component by EDFA or SOA3. Furthermore, the ambiguity in the broad smooth background below the coherence spike probably indicates that the retrieved pulse train is variably spaced. The coherence spike results from the short coherent component of the pulse train, while the background pedestal results from the overall average pulse length [23].

Another set of AC traces and optical spectra are shown in Figs. 5(b) and 5(a). The bias condition is $V_{\mathrm{SA}}=-1.1 \mathrm{~V}$, $I_{\mathrm{GAIN}}=150 \mathrm{~mA}, I_{\mathrm{BOOST}}=50 \mathrm{~mA}$. The $3-\mathrm{dB}$ bandwidth

(a)

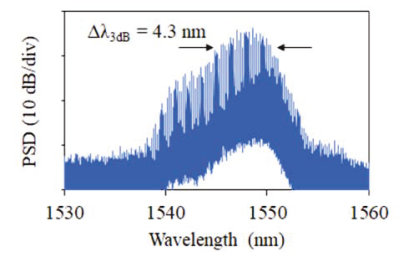

(b)

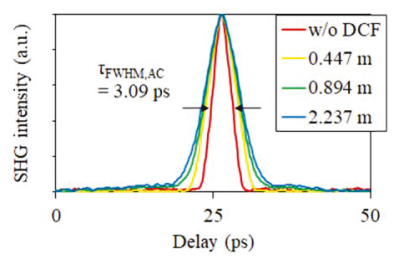

Fig. 5. At Out2, $V_{\mathrm{SA}}=-1.1 \mathrm{~V}, \quad I_{\mathrm{GAIN}}=150 \mathrm{~mA}$, $I_{\text {BOOST }}=50 \mathrm{~mA}$. (a) Optical spectrum. 3-dB bandwidth $=$ $4.3 \mathrm{~nm}(540 \mathrm{GHz})$. (b) Autocorrelation trace with different DCF lengths. $\tau_{\mathrm{FWHM}, \mathrm{AC}}=3.09$ ps. Assuming a sech2 pulse shape, $\tau_{\mathrm{FWHM}, \mathrm{SECH} 2}=2.01 \mathrm{ps}$.

(a)

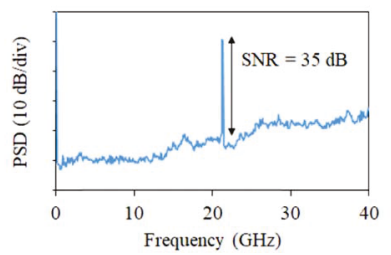

(b)

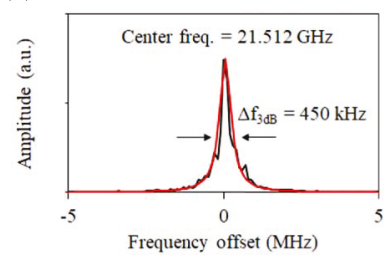

Fig. 6. (a) RF spectrum when $V_{\mathrm{SA}}=-0.6 \mathrm{~V}, I_{\mathrm{GAIN}}=180 \mathrm{~mA}$, $I_{\mathrm{BOOST}}=30 \mathrm{~mA}$. SNR is $35 \mathrm{~dB}$. (b) RF spectrum with same bias condition. RF linewidth is $450 \mathrm{kHz}$.

is $4.3 \mathrm{~nm}(540 \mathrm{GHz})$, much narrower than that of Fig. 4(a). For evaluating the dispersion of the overall optical path including the PIC under test, lensed fiber, and EDFA, three dispersion compensating fibers (DCF) were used, as shown in Fig. 5(b). When no DCF is inserted, the AC FWHM duration is 3.09 ps. Assuming a sech ${ }^{2}$ shape, the pulse width is 2.01 ps. The duration increases as a longer DCF is applied, confirming that the accumulated dispersion is not compensated by the DCFs. Despite this, these AC traces are pedestal-free, from which the retrieved pulse train is more stable than that in Fig. 4(b). The TBP is 1.08 .

The RF spectrum of the beat note through the $40-\mathrm{GHz}$ photodiode is demonstrated in Fig. 6. Figure 6(a) shows the full span up to $40 \mathrm{GHz}$ with $1-\mathrm{MHz}$ resolution bandwidth, while Fig. 6(b) shows the RF tone in detail with a $3-\mathrm{kHz}$ resolution bandwidth. In Fig. 6(a), the RF tone signal is at $21.5 \mathrm{GHz}$, and the $\mathrm{SNR}$ is about $35 \mathrm{~dB}$. The clean and strong fundamental tone that is $35 \mathrm{~dB}$ above any spurious peaks or noise floor indicates that there is little residual amplitude. In Fig. 6(b), the RF tone is observed at $21.512 \mathrm{GHz}$ with a linewidth of $450 \mathrm{kHz}$.

We have presented a record broad $(14 \mathrm{~nm}, \sim 1.8 \mathrm{THz}$ at 3 -dB level) optical frequency comb generated from a passively mode-locked QW laser. This 21.5-GHz colliding-pulse modelocked laser with intracavity phase modulators was developed in an InP generic photonic integration technology platform. By inserting a booster amplifier SOA, higher power $>1 \mathrm{~mW}$ has been achieved. The autocorrelation trace and RF spectrum have agreed with the mode-locking scheme, exhibiting a narrow pulse width of 0.35 ps on a pedestal as well as an RF linewidth of $450 \mathrm{kHz}$ and a $35-\mathrm{dB}$ SNR.

\section{Funding}

Horizon 2020 Framework Programme (H2020)(642355); Spanish Ministerio de Economía y Competitividad (MINECO) DiDACTIC (TEC2013-47753-C3-3-R); Consejería de Educación, Juventud y Deporte of Comunidad de Madrid (P2013/ICE-3004).

\section{Acknowledgment}

We thank Dr. Julien Javaloyes and Prof. Elliott Brown for helpful discussions. 


\section{REFERENCES}

1. P. J. Delfyett, S. Gee, M. T. Choi, H. Izadpanah, W. Lee, S. Ozharar, F. Quinlan, and T. Yilmaz, J. Lightwave Technol. 24, 2701 (2006).

2. J. Javaloyes and S. Balle, IEEE J. Quantum Electron. 46, 1023 (2010).

3. E. A. Avrutin, J. H. Marsh, and E. L. Portnoi, IEE Proc. J. Optoelectron. 147, 251 (2000).

4. M. Smit, X. Leijtens, E. Bente, J. Van der Tol, H. Ambrosius, D. Robbins, M. Wale, N. Grote, and M. Schell, IET Optoelectron. 5, 187 (2011).

5. R. G. M. P. Koumans and R. Van Roijen, IEEE J. Quantum Electron. 32, 478 (1996).

6. K. A. Williams, M. G. Thompson, and I. H. White, New J. Phys. 6, 179 (2004).

7. M. S. Tahvili, Y. Barbarin, X. J. M. Leijtens, T. de Vries, E. Smalbrugge, J. Bolk, H. P. M. M. Ambrosius, M. K. Smit, and E. A. J. M. Bente, Opt. Lett. 36, 2462 (2011).

8. E. Bente, V. Moskalenko, S. Latkowski, S. Tahvili, L. Augustin, and M. Smit, Proc. SPIE 9134, 91340C (2014).

9. C. Gordón, R. Guzmán, V. Corral, M.-C. Lo, and G. Carpintero, J. Lightwave Technol. 34, 4722 (2016).

10. M.-C. Lo, R. Guzmán, C. Gordón, and G. Carpintero, Opt. Lett. 42, 1532 (2017).

11. J. S. Parker, A. Bhardwaj, P. R. A. Binetti, Y.-J. Hung, and L. A. Coldren, IEEE Photon. Technol. Lett. 24, 131 (2012).

12. V. Moskalenko, J. Javaloyes, S. Balle, M. K. Smit, and E. A. J. M. Bente, IEEE J. Quantum Electron. 50, 415 (2014).

13. V. Corral, R. Guzmán, C. Gordón, X. J. M. Leijtens, and G. Carpintero, Opt. Lett. 41, 1937 (2016).

14. M. G. Thompson, A. Rae, X. Mo, R. V. Penty, and I. H. White, IEEE J. Sel. Top. Quantum Electron. 15, 661 (2009).

15. G.-H. Duan, A. Shen, A. Akrout, F. V. Dijk, F. Lelarge, F. Pommereau, O. LeGouezigou, J.-G. Provost, H. Gariah, F. Blache, F. Mallecot, K Merghem, A. Martinez, and A. Ramdane, Bell Labs Tech. J. 14, 63 (2009).

16. R. Rosales, K. Merghem, A. Martinez, A. Akrout, J.-P. Tourrenc, A. Accard, F. Lelarge, and A. Ramdane, IEEE J. Sel. Top. Quantum Electron. 17, 1292 (2011).

17. R. Rosales, S. G. Murdoch, R. T. Watts, K. Merghem, A. Martinez, F. Lelarge, A. Accard, L. P. Barry, and A. Ramdane, Opt. Express 20 8649 (2012).

18. V. Moskalenko, S. Latkowski, S. Tahvili, T. de Vries, M. Smit, and E. Bente, Opt. Express 22, 28865 (2014).

19. V. Moskalenko, J. Koelemeij, K. Williams, and E. Bente, Opt. Lett. 42 , 1428 (2017)

20. SMART Photonics B. V., http://www.smartphotonics.nl/.

21. Joint European Platform for Photonic Integration of Components and Circuits, http://www.jeppix.eu/.

22. C. Gordón, R. Guzmán, V. Corral, X. Leijtens, and G. Carpintero, Opt. Express 23, 14666 (2015).

23. M. Rhodes, G. Steinmeyer, J. Ratner, and R. Trebino, Laser Photon. Rev. 7, 557 (2013). 